\title{
Risk factors for Hyperhomocysteinemia for Specific MTHFR C677T Genotypes and Gender in Chinese Population.
}

\section{Hang Xiang ( $\nabla$ mailbox9999@126.com )}

Chinese PLA General Hospital https://orcid.org/0000-0003-2764-4458

\section{Tianyuan Xiang}

Chinese PLA General Hospital

\section{Muyang Yan \\ Chinese PLA General Hospital}

\section{Sheng Yu}

Southern Medical University Nanfang Hospital

Matthew John Horwedel

Brigham and Women's Hospital

\section{Qiang Zeng}

Chinese PLA General Hospital

\section{Yang Li}

Chinese PLA General Hospital

\section{Research article}

Keywords: Gender, Hyperhomocysteine, MTHFR polymorphism, Risk factor

Posted Date: August 17th, 2020

DOl: https://doi.org/10.21203/rs.3.rs-57500/v1

License: (1) (1) This work is licensed under a Creative Commons Attribution 4.0 International License. Read Full License 


\section{Abstract}

Background--Our previous studies have identified that both gender and genotype of MTHFR C677T were independent variables for plasma homocysteine (Hcy) levels. Based on these results, we want to further explore their systemic information, in order to find specific risk factors for each genetic group, which might be used as predictors or targeting markers for lowering Hcy levels.

Patients and Methods-This cross section study was performed through November 2017 to July 2019. A total of 4534 adults aged 20-75y were selected for this study, and all of them underwent a physical examinations and MTHFR C677T genotyping.

Results-The average of Hcy level was higher in TT genotype than CC and CT genotypes ( $P=0.000)$. Multiple linear regression analysis found that except the common protective factors (folate and Vit B12) and risk factor ( $\mathrm{Cr}$ ), each group has it specific risk factors for HHcy-female-CT (age, SBP and $\mathrm{Hb}$ ), femaleTT (SBP and AST); male-CC (age, AST and Hb), male-CT (age and AST) and male-TT (SBP, AST and Hb).

Conclusion-The plasma Hcy level was influenced by different risk factors for specific gender and genotype. These risk factors might be useful for prediction or prevention of HHcy in the future.

\section{Introduction}

Hyperhomocysteinemia (HHcy) (1), which is defined as a moderately elevated plasm Hcy concentration, has been demonstrated as an independent risk factor for hypertension, stroke and cardiovascular disease $(2-5)$. Therefore, serum Hcy level became a potential therapeutic target for preventing or alleviating cardioand cerebral-vascular disease (6). Physiologically, the metabolism of Hcy is regulated by a pivotal enzyme named MTHFR, which is a flavin adenine dinucleotide (coenzyme of riboflavin)-dependent enzyme, that catalyzing N5-N10 methylene tetrahydrofolate to N5-methyl tetrahydrofolate (7). The mutation of MTHFR C677T will decrease the enzyme activity (8), and eventually leading to a significant elevation of Hcy concentrations $(9,10,11)$.

The aim of this study was to explore the risk factors for HHcy, which might be used as predictors or targeting markers for HHcy. Before this study, there have been several studies which also targeting for exploring risk factors for HHcy $(12,14)$, but they were not stratified by genders or genotypes $(12,14)$. Our previous studies have found that both gender and genotype of MTHFR C677T were independent variables for plasma Hcy levels. $(12,15)$. And food consumption would pose different influence on Hcy levels for each gender and genotype (13). Based on these results, we supposed that each gender and genotype may have their specific risk factors for HHcy.

\section{Patients And Methods}

\section{Study Design and Ethics Approval}


The Ethics Committee at PLA General Hospital approved the protocol of this cross-sectional, observational, non randomized study, which was designed in accordance with the principle of the Declaration of Helsinki (clinical trial ID: S2016-098-02). All subjects gave their informed consent to participate in the study. The study was carried out at the Health Management Institute of Chinese PLA General Hospital during the period of November 2017 to July 2019.

Written, informed consent was obtained from all patients to publish their innominate data.

\section{Subjects}

A total of 4770 participants who completed a health examination, were enrolled in this study. The inclusion criteria were: aged between 20 and 80 , not taking folic acid supplements or using agents that affect vitamin $\mathrm{B}$ and folic acid metabolism, such as methotrexate and anticonvulsants, and being free from folic medicine for at least 6 months.

Among those participants, 34 participants with serious renal disease, 54 participants with hepatic disease, and 123 participants with hypothyroidism were excluded from the study. In addition, 25 participants were excluded for the inaccuracy of the results. In all, 1369 females and 3165 males were selected for studying in this cross section study.

\section{Outcome Measures}

Assessment of MTHFR C677T Genotype. Genetic polymorphisms MTHFR $677 \mathrm{C} \rightarrow$ T were detected using gene chip hybrid analysis. Genomic DNA was extracted from the whole blood of the participants using the QIAamp ${ }^{\circledR}$ DNA Mini Kit (CAT No. 51304, Germany). The PCR, hybridization, gene array detection and analysis were conducted strictly according to the manuals of the BaiO genotype detecting gene array kit and equipment (BaiO Technology Corp).

Assessment of Covariate. The patients were subjected to a health examination, including height and weight measurements and blood pressure. Fasting blood was extracted for detection. Plasma Hcy was analyzed by HPLC with fluorometric detection $(16,17)$. Folate concentration was measured using a dual count Solid Phase Boil Radio assay (Diagnostic Products, Los Angeles, CA). The vitamin (Vit) B12 was detected by liquid chromatography-tandem mass spectrometry (18). The blood glucose levels were measured using the hexokinase method; serum levels of bilirubin, Alanine aminotransferase (ALT) and Asparagine aminotransferase (AST) were measured using a BM Hitachi 711 Chemistry Analyzer. The total cholesterol (TC), high-density lipoprotein (HDL), low-density lipoprotein (LDH) and TG levels were measured with a colorimetric method (Cobas c 501 autoanalyzer, Roche Diagnostics, Germany). Serum $\mathrm{Cr}$ and UA were measured by a modified kinetic rate Jaffe reaction method using a Dade Dimension Chemistry Analyzer (Siemens). Cancer biomarkers of CEA and AFP were measured using electrochemiluminescence immunoassay. As the baseline of the Hcy level was remarkably different 
between sexes, HHcy was define distinctively for male ( $\geqq 15 \mu \mathrm{mol} / \mathrm{L})$ and female $(\geqq 10 \mu \mathrm{mol} / \mathrm{L})(12,14)$. Standard quality control procedures were performed each day with standard samples (CV $<10 \%)$.

\section{Statistical Analysis}

Participants were grouped by genders and genotypes. Comparison of the variables was assessed using an ANOVA or Chi-square test. Multiple linear regression analyses were performed between Hcy and the variables with stepwise methods. The standard coefficients for the variable were used to estimate the strength of the association. All tests were two-tailed, and $P$ values less than 0.05 were considered to indicate significant differences. The statistical analyses were carried out using SPSS version 17.0 and SAS version 8.02 or 9.1 .

\section{Results}

\section{Clinical Characteristics}

There were 4534 individuals selected for this study, including 3165 males (CC $=724, C T=1523$ and TT $=$ 918) and 1369 females $(C C=320, C T=683$ and TT = 366). There were significant difference between

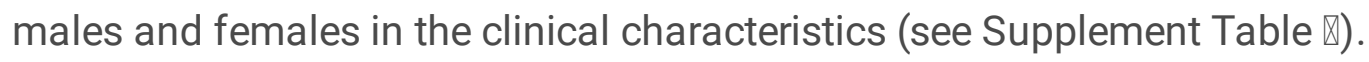

Further analysis was performed in each gender group and stratified by genotypes. For males, Hcy level was significantly different between 3 genotypes with the mean values of $12.30 \pm 3.34 \mu \mathrm{mol}, 12.94 \pm$ $4.57 \mu \mathrm{mol}$ and $19.37 \pm 9.26 \mu \mathrm{mol}$ for male-CC, male-CT and male-TT, respectively. And other factors were also identified with statistical significance, such as folate, Vit B12, HDL, TG and height $(p<0.05)$.

For females, the mean values of Hcy level for female-CC, female-CT, female-TT were $8.85 \pm 3.53 \mu \mathrm{mol}$, $9.22 \pm 3.34 \mu \mathrm{mol}$ and $11.39 \pm 4.98 \mu \mathrm{mol}$, respectively. And significant difference existed in the Hcy, folate, Vit B12, G2h and HDL between the female 3 genotype groups $(P<0.05)$ (see Table 1). 
Table 1

Clinical characteristics of the participants for each gender and genotype.

\begin{tabular}{|c|c|c|c|c|c|c|c|c|}
\hline \multirow[t]{2}{*}{ Variables } & \multicolumn{4}{|l|}{ Male } & \multicolumn{4}{|l|}{ Female } \\
\hline & $\begin{array}{l}C C(n= \\
726)\end{array}$ & $\begin{array}{l}C T(n= \\
1523)\end{array}$ & $\begin{array}{l}\text { TT(n = } \\
918)\end{array}$ & $\mathrm{P}+$ & $\begin{array}{l}C C(n= \\
320)\end{array}$ & $\begin{array}{l}\text { CT }(n= \\
683)\end{array}$ & $\begin{array}{l}\operatorname{TT}(\mathrm{n}= \\
366)\end{array}$ & $\mathrm{P}+$ \\
\hline Stroke (n) & 6 & 13 & 10 & 0.75 & 3 & 3 & 2 & - \\
\hline $\mathrm{CHD}(\mathrm{n})$ & 27 & 78 & 55 & 0.12 & 15 & 20 & 16 & 0.20 \\
\hline $\operatorname{HBP}(n)$ & 245 & 468 & 318 & 0.07 & 51 & 98 & 72 & 0.11 \\
\hline $\operatorname{Age}(y)$ & $\begin{array}{l}48.83 \\
\pm 7.76\end{array}$ & $\begin{array}{l}49.09 \\
\pm 7.23\end{array}$ & $\begin{array}{l}49.22 \\
\pm 7.51\end{array}$ & 0.58 & $\begin{array}{l}48.95 \pm \\
8.65\end{array}$ & $\begin{array}{l}48.28 \pm \\
8.73\end{array}$ & $\begin{array}{l}50.19 \\
\pm 8.49\end{array}$ & 0.94 \\
\hline Height(cm) & $\begin{array}{l}172.76 \\
\pm 6.07\end{array}$ & $\begin{array}{l}173.21 \\
\pm 5.66\end{array}$ & $\begin{array}{l}173.41 \\
\pm 5.72\end{array}$ & 0.04 & $\begin{array}{l}160.95 \pm \\
5.68\end{array}$ & $\begin{array}{l}161.61 \pm \\
5.25\end{array}$ & $\begin{array}{l}161.20 \\
\pm 5.15\end{array}$ & 0.10 \\
\hline Weight(kg) & $\begin{array}{l}78.24 \\
\pm 11.34\end{array}$ & $\begin{array}{l}78.74 \\
\pm 10.53\end{array}$ & $\begin{array}{l}79.42 \\
\pm 10.25\end{array}$ & 0.08 & $\begin{array}{l}61.56 \pm \\
8.39\end{array}$ & $\begin{array}{l}61.55 \pm \\
8.85\end{array}$ & $\begin{array}{l}62.20 \\
\pm 8.84\end{array}$ & 0.77 \\
\hline BMI & $\begin{array}{l}26.13 \\
\pm 3.37\end{array}$ & $\begin{array}{l}26.23 \\
\pm 3.64\end{array}$ & $\begin{array}{l}26.38 \\
\pm 3.18\end{array}$ & 0.38 & $\begin{array}{l}23.81 \pm \\
3.40\end{array}$ & $\begin{array}{l}23.56 \pm \\
3.15\end{array}$ & $\begin{array}{l}23.92 \\
\pm 3.14\end{array}$ & 0.38 \\
\hline $\mathrm{WC}(\mathrm{cm})$ & $\begin{array}{l}92.34 \\
\pm 9.21\end{array}$ & $\begin{array}{l}92.69 \\
\pm 8.35\end{array}$ & $\begin{array}{l}93.01 \\
\pm 8.58\end{array}$ & 0.42 & $\begin{array}{l}80.26 \pm \\
9.21\end{array}$ & $\begin{array}{l}79.92 \pm \\
9.01\end{array}$ & $\begin{array}{l}81.49 \\
\pm 9.09\end{array}$ & 0.97 \\
\hline $\mathrm{SBP}(\mathrm{mmHg})$ & $\begin{array}{l}120.54 \\
\pm 17.24\end{array}$ & $\begin{array}{l}121.90 \\
\pm 16.72\end{array}$ & $\begin{array}{l}121.46 \\
\pm 16.23\end{array}$ & 0.44 & $\begin{array}{l}111.18 \pm \\
18.56\end{array}$ & $\begin{array}{l}109.49 \pm \\
19.18\end{array}$ & $\begin{array}{l}111.65 \\
\pm 19.47\end{array}$ & 0.58 \\
\hline $\mathrm{DBP}(\mathrm{mmHg})$ & $\begin{array}{l}79.44 \\
\pm 11.34\end{array}$ & $\begin{array}{l}79.52 \\
\pm 11.42\end{array}$ & $\begin{array}{l}79.72 \\
\pm 11.45\end{array}$ & 0.78 & $\begin{array}{l}74.40 \pm \\
10.06\end{array}$ & $\begin{array}{l}73.43 \pm \\
11.52\end{array}$ & $\begin{array}{l}74.96 \\
\pm 11.64\end{array}$ & 0.62 \\
\hline $\operatorname{Hcy}(\mu \mathrm{mol} / \mathrm{L})$ & $\begin{array}{l}12.30 \\
\pm 3.34\end{array}$ & $\begin{array}{l}12.94 \\
\pm 4.57\end{array}$ & $\begin{array}{l}19.37 \\
\pm 9.26\end{array}$ & $0.00 *$ & $\begin{array}{l}8.85 \pm \\
3.53\end{array}$ & $\begin{array}{l}9.22 \pm \\
3.34\end{array}$ & $\begin{array}{l}11.39 \\
\pm 4.98\end{array}$ & $0.00 *$ \\
\hline $\begin{array}{l}\text { Folate } \\
(\mathrm{ng} / \mathrm{mL})\end{array}$ & $\begin{array}{l}10.23 \\
\pm 3.32\end{array}$ & $\begin{array}{l}9.37 \pm \\
3.46\end{array}$ & $\begin{array}{l}8.28 \pm \\
3.36\end{array}$ & $0.00 *$ & $\begin{array}{l}11.63 \pm \\
3.70\end{array}$ & $\begin{array}{l}10.29 \pm \\
3.89\end{array}$ & $\begin{array}{l}9.12 \pm \\
3.69\end{array}$ & $0.03 *$ \\
\hline $\begin{array}{l}\text { Vit } \\
\text { B12(pg/ml) }\end{array}$ & $\begin{array}{l}584.48 \\
\pm \\
244.87\end{array}$ & $\begin{array}{l}569.36 \\
\pm \\
236.35\end{array}$ & $\begin{array}{l}525.85 \\
\pm \\
225.36\end{array}$ & $0.00 *$ & $\begin{array}{l}625.95 \pm \\
275.72\end{array}$ & $\begin{array}{l}605.19 \pm \\
284.77\end{array}$ & $\begin{array}{l}589.72 \\
\pm \\
284.14\end{array}$ & $0.02 *$ \\
\hline $\mathrm{Hb}(\mathrm{g} / \mathrm{L})$ & $\begin{array}{l}152.31 \\
\pm 10.74\end{array}$ & $\begin{array}{l}154.19 \\
\pm 10.32\end{array}$ & $\begin{array}{l}154.50 \\
\pm 9.97\end{array}$ & 0.79 & $\begin{array}{l}130.71 \pm \\
12.70\end{array}$ & $\begin{array}{l}130.59 \pm \\
11.91\end{array}$ & $\begin{array}{l}130.96 \\
\pm 11.81\end{array}$ & 0.55 \\
\hline $\operatorname{ALT}(\mathrm{U} / \mathrm{L})$ & $\begin{array}{l}28.68 \\
\pm 20.66\end{array}$ & $\begin{array}{l}28.48 \\
\pm 22.72\end{array}$ & $\begin{array}{l}27.53 \\
\pm 16.65\end{array}$ & 0.64 & $\begin{array}{l}16.69 \pm \\
10.17\end{array}$ & $\begin{array}{l}17.77 \pm \\
11.45\end{array}$ & $\begin{array}{l}18.14 \\
\pm 12.62\end{array}$ & 0.26 \\
\hline
\end{tabular}

Note: HBP: hypertension; CHD: coronary heart disease; BMI: body mass index; WC: Waist circumference; SBP: systolic blood pressure; DBP: diastolic blood pressure; Hcy: homocysteine; Hb: hemoglobin; FPG: fasting plasma glucose; G2h: postprandial 2 hours blood glucose; TC: Total cholesterol; TG: triglyceride; CRP: C-reactive protein; ALT: Alanine aminotransferase; AST: Aspartate transaminase; LDL-C: Low-density lipoprotein; HDL-C: high-density lipoprotein; AFP:alpha fetoprotein; CEA:carcinoembryonicantigen; UA: Blood uric acid; Cr: Blood creatinine. Pt: Comparisions between genotypes. ${ }^{*} \mathrm{P}<0.05$. 


\begin{tabular}{|c|c|c|c|c|c|c|c|c|}
\hline \multirow{2}{*}{$\begin{array}{l}\text { Variables } \\
\text { AST(U/L) }\end{array}$} & \multicolumn{3}{|l|}{ Male } & \multicolumn{3}{|c|}{ Female } & \multirow[b]{2}{*}{$\begin{array}{l}18.78 \\
\pm 6.92\end{array}$} & \multirow[b]{2}{*}{0.49} \\
\hline & $\begin{array}{l}23.45 \\
\pm 11.34\end{array}$ & $\begin{array}{l}22.64 \\
\pm 21.81\end{array}$ & $\begin{array}{l}21.85 \\
\pm 8.89\end{array}$ & 0.43 & $\begin{array}{l}18.11 \pm \\
5.52\end{array}$ & $\begin{array}{l}18.52 \pm \\
6.318\end{array}$ & & \\
\hline $\begin{array}{l}\text { FPG } \\
(\mathrm{mmol} / \mathrm{L})\end{array}$ & $\begin{array}{l}5.88 \pm \\
1.62\end{array}$ & $\begin{array}{l}5.89 \pm \\
1.53\end{array}$ & $\begin{array}{l}5.93 \pm \\
1.70\end{array}$ & 0.79 & $\begin{array}{l}5.28 \pm \\
0.77\end{array}$ & $\begin{array}{l}5.28 \pm \\
0.94\end{array}$ & $\begin{array}{l}5.32 \pm \\
0.81\end{array}$ & 0.82 \\
\hline $\begin{array}{l}\mathrm{G} 2 \mathrm{~h} \\
(\mathrm{mmol} / \mathrm{L})\end{array}$ & $\begin{array}{l}7.55 \pm \\
2.78\end{array}$ & $\begin{array}{l}7.63 \pm \\
2.73\end{array}$ & $\begin{array}{l}7.70 \pm \\
2.82\end{array}$ & 0.57 & $\begin{array}{l}7.38 \pm \\
2.16\end{array}$ & $\begin{array}{l}7.18 \pm \\
2.02\end{array}$ & $\begin{array}{l}7.53 \pm \\
1.90\end{array}$ & $0.04^{\star}$ \\
\hline $\mathrm{TC}(\mathrm{mmol} / \mathrm{L})$ & $\begin{array}{l}4.77 \pm \\
0.92\end{array}$ & $\begin{array}{l}4.75 \pm \\
0.89\end{array}$ & $\begin{array}{l}4.73 \pm \\
0.90\end{array}$ & 0.71 & $\begin{array}{l}4.77 \pm \\
0.94\end{array}$ & $\begin{array}{l}4.72 \pm \\
0.93\end{array}$ & $\begin{array}{l}4.76 \pm \\
0.91\end{array}$ & 0.89 \\
\hline $\mathrm{TG}(\mathrm{mmol} / \mathrm{L})$ & $\begin{array}{l}2.12 \pm \\
1.95\end{array}$ & $\begin{array}{l}2.13 \pm \\
1.86\end{array}$ & $\begin{array}{l}2.15 \pm \\
1.98\end{array}$ & $0.04^{*}$ & $\begin{array}{l}1.33 \pm \\
0.90\end{array}$ & $\begin{array}{l}1.32 \pm \\
0.98\end{array}$ & $\begin{array}{l}1.26 \pm \\
0.70\end{array}$ & 0.14 \\
\hline $\begin{array}{l}\text { HDL- } \\
\text { C(mmol/L) }\end{array}$ & $\begin{array}{l}1.14 \pm \\
0.30\end{array}$ & $\begin{array}{l}1.11 \pm \\
0.28\end{array}$ & $\begin{array}{l}1.11 \pm \\
0.28\end{array}$ & $0.05^{\star}$ & $\begin{array}{l}1.44 \pm \\
0.37\end{array}$ & $\begin{array}{l}1.39 \pm \\
0.34\end{array}$ & $\begin{array}{l}1.40 \pm \\
0.34\end{array}$ & $0.02^{*}$ \\
\hline $\begin{array}{l}\text { LDL- } \\
\mathrm{C}(\mathrm{mmol} / \mathrm{L})\end{array}$ & $\begin{array}{l}3.06 \pm \\
0.84\end{array}$ & $\begin{array}{l}3.11 \pm \\
0.78\end{array}$ & $\begin{array}{l}3.10 \pm \\
0.76\end{array}$ & 0.89 & $\begin{array}{l}3.08 \pm \\
0.84\end{array}$ & $\begin{array}{l}3.08 \pm \\
0.86\end{array}$ & $\begin{array}{l}3.12 \pm \\
0.82\end{array}$ & 0.57 \\
\hline $\operatorname{AFP}(\mu \mathrm{g} / \mathrm{L})$ & $\begin{array}{l}3.23 \pm \\
8.00\end{array}$ & $\begin{array}{l}3.10 \pm \\
2.10\end{array}$ & $\begin{array}{l}3.14 \pm \\
1.67\end{array}$ & 0.74 & $\begin{array}{l}2.60 \pm \\
1.55\end{array}$ & $\begin{array}{l}2.72 \pm \\
1.64\end{array}$ & $\begin{array}{l}2.76 \pm \\
1.75\end{array}$ & 0.63 \\
\hline $\mathrm{CEA}(\mu \mathrm{g} / \mathrm{mL})$ & $\begin{array}{l}1.94 \pm \\
1.23\end{array}$ & $\begin{array}{l}1.96 \pm \\
1.29\end{array}$ & $\begin{array}{l}2.00 \pm \\
1.52\end{array}$ & 0.82 & $\begin{array}{l}1.29 \pm \\
0.95\end{array}$ & $\begin{array}{l}1.19 \pm \\
0.84\end{array}$ & $\begin{array}{l}1.22 \pm \\
0.84\end{array}$ & 0.18 \\
\hline $\mathrm{UA}(\mu \mathrm{mol} / \mathrm{L})$ & $\begin{array}{l}382.52 \\
\pm 78.47\end{array}$ & $\begin{array}{l}378.79 \\
\pm 74.02\end{array}$ & $\begin{array}{l}375.24 \\
\pm 75.88\end{array}$ & 0.17 & $\begin{array}{l}265.54 \pm \\
58.26\end{array}$ & $\begin{array}{l}268.41 \pm \\
56.63\end{array}$ & $\begin{array}{l}264.69 \\
\pm 54.15\end{array}$ & 0.33 \\
\hline $\mathrm{Cr}(\mu \mathrm{mol} / \mathrm{L})$ & $\begin{array}{l}74.20 \\
\pm 11.48\end{array}$ & $\begin{array}{l}74.15 \\
\pm 12.81\end{array}$ & $\begin{array}{l}73.68 \\
\pm 14.69\end{array}$ & 0.63 & $\begin{array}{l}54.51 \pm \\
8.70\end{array}$ & $\begin{array}{l}54.85 \pm \\
8.17\end{array}$ & $\begin{array}{l}54.59 \\
\pm 8.57\end{array}$ & 0.97 \\
\hline \multicolumn{9}{|c|}{$\begin{array}{l}\text { Note: HBP: hypertension; CHD: coronary heart disease; BMI: body mass index; WC: Waist } \\
\text { circumference; SBP: systolic blood pressure; DBP: diastolic blood pressure; Hcy: homocysteine; Hb: } \\
\text { hemoglobin; FPG: fasting plasma glucose; G2h: postprandial } 2 \text { hours blood glucose; TC: Total } \\
\text { cholesterol; TG: triglyceride; CRP: C-reactive protein; ALT: Alanine aminotransferase; AST: Aspartate } \\
\text { transaminase; LDL-C: Low-density lipoprotein; HDL-C: high-density lipoprotein; AFP:alpha fetoprotein; } \\
\text { CEA:carcinoembryonicantigen; UA: Blood uric acid; Cr: Blood creatinine. Pt: Comparisions between } \\
\text { genotypes. *P < } 0.05 \text {. }\end{array}$} \\
\hline
\end{tabular}

\section{The Risk Factors for HHcy in Each Gender and Genotype}

In the males, after adjustment of weight, height, BMI and other variables, we found that folate, VitB12 were negatively correlated with HHcy, while Cr were positively correlated with HHcy. And genotype (CC and CT) was a protective factor for HHcy (see Supplement Table II). After stratified by genotypes, we found specific positive risk factors for each genotype, even though they have some common negative factors, such as folate and VitB12. In male CC group, age, AST and $\mathrm{Hb}$ were positive risk factors for HHcy; In the male CT group, age and AST were positive risk factors for HHcy; In the male TT group, SBP, AST and Hb were positive risk factors for HHcy (see Table 2). 
Table 2

Multilinear regression for the risk factors of HHcy for each genotype in males.

\begin{tabular}{|c|c|c|c|c|c|}
\hline Gender-Genotype & Variables & B & standard error & standardized coefficient & $P$ value \\
\hline \multicolumn{6}{|l|}{$\mathrm{M}-\mathrm{CC}$} \\
\hline & Folate & -.265 & .033 & -.289 & .000 \\
\hline & VitB12 & -.003 & .000 & -.211 & .000 \\
\hline & Age & .065 & .015 & .159 & .000 \\
\hline & AST & .054 & .017 & .202 & .001 \\
\hline & $\mathrm{HGB}$ & .032 & .011 & .105 & .005 \\
\hline & $\mathrm{Cr}$ & .047 & .010 & .166 & .000 \\
\hline \multicolumn{6}{|c|}{ ANOVA: $F=25.370, P=0.000 ; R 2=0.248$, adjusted $R 2=0.238$} \\
\hline \multicolumn{6}{|l|}{ M-CT } \\
\hline & (Constant) & 9.563 & 1.445 & & .000 \\
\hline & Folate & -.412 & .034 & -.310 & .000 \\
\hline & VitB12 & -.005 & .001 & -.230 & .000 \\
\hline & Age & .038 & .016 & .059 & .020 \\
\hline & AST & .011 & .005 & .057 & .025 \\
\hline & $\mathrm{Cr}$ & .080 & .009 & .225 & .000 \\
\hline \multicolumn{6}{|c|}{ ANOVA: $F=51.008, P=0.000 ; R 2=0.250$, adjusted $R 2=0.245$} \\
\hline \multicolumn{6}{|l|}{ M-TT } \\
\hline & (Constant) & 10.353 & 5.130 & & .044 \\
\hline & Folate & -.991 & .081 & -.375 & .000 \\
\hline & VitB12 & -.011 & .001 & -.269 & .000 \\
\hline & SBP & .038 & .016 & .069 & .018 \\
\hline & HGB & .087 & .029 & .088 & .003 \\
\hline & AST & .085 & .030 & .084 & .004 \\
\hline & $\mathrm{Cr}$ & .106 & .018 & .171 & .000 \\
\hline \multicolumn{6}{|c|}{ ANOVA: $F=53.868, P=0.000 ; R 2=0.354$, adjusted $R 2=0.347$} \\
\hline \multicolumn{6}{|c|}{ Table 3. Multilinear regression analysis for the risk factors of HHcy for each genotype in females. } \\
\hline Gender-Genotype & Variables & B & standard error & standardized coefficient & $P$ value \\
\hline
\end{tabular}




\begin{tabular}{|c|c|c|c|c|c|}
\hline Gender-Genotype & Variables & B & standard error & standardized coefficient & $P$ value \\
\hline \multicolumn{6}{|l|}{ F-CC } \\
\hline & (Constant) & 7.154 & 2.423 & & .003 \\
\hline & Folate & -.119 & .053 & -.125 & .027 \\
\hline & VitB12 & -.004 & .001 & -.333 & .000 \\
\hline & $\mathrm{Cr}$ & .083 & .022 & .206 & .000 \\
\hline \multicolumn{6}{|c|}{ ANOVA: $F=14.396, P=0.000 ; R^{2}=0.281$, adjusted $R^{2}=0.261$} \\
\hline \multicolumn{6}{|l|}{ F-CT } \\
\hline & Folate & -.172 & .033 & -.201 & .000 \\
\hline & VitB12 & -.004 & .000 & -.330 & .000 \\
\hline & Age & .062 & .017 & .158 & .000 \\
\hline & SBP & .015 & .007 & .088 & .033 \\
\hline & HGB & .027 & .011 & .095 & .014 \\
\hline & $\mathrm{Cr}$ & .075 & .015 & .180 & .000 \\
\hline \multicolumn{6}{|c|}{ ANOVA: $F=35.463, P=0.000 ; R^{2}=0.282$, adjusted $R^{2}=0.274$} \\
\hline \multicolumn{6}{|l|}{ F-TT } \\
\hline & (Constant) & 5.058 & 2.064 & & .015 \\
\hline & Folate & -.453 & .063 & -.333 & .000 \\
\hline & VitB12 & -.005 & .001 & -.273 & .000 \\
\hline & SBP & .046 & .011 & .185 & .000 \\
\hline & AST & .108 & .032 & .150 & .001 \\
\hline & $\mathrm{Cr}$ & .124 & .025 & .218 & .000 \\
\hline \multicolumn{6}{|c|}{ ANOVA: $F=33.856, P=0.000 ; R^{2}=0.336$, adjusted $R^{2}=0.326$} \\
\hline
\end{tabular}

In the females, we also found negative risk factors (genotype, folate and VitB12) and positive risk factor (Cr), after adjustment of weight, height, BMI and other variables (see Supplement Table II). We also found specific risk factors after stratified by genotype, though they share the negative factors of folate and VitB12. In the female CT group, one positive risk factor $(\mathrm{Cr})$ was identified. In female CT group, 4 positive risk factor (age, SBP, $\mathrm{Hb}$ and $\mathrm{Cr}$ ) were correlated with Hcy level. In female TT group, 3 positive risk factors (SBP, AST and Cr) were found to be correlated with Hcy level (see Table 3). 


\section{Discussion}

HHcy has been proven to be independent risk factors for hypertension and stroke (19). People have found several factors that would cause HHcy, such as MTHFR C677T polymorphism, folate and vitamin B (2022). At present, it is recommended that Hcy concentration should be lowered by supplement of folate and Vit B12 through food nutrition (23) or Synthetic medicine (24). However, this intervention did not show equal effects in all the populations-it is most effective in TT genotype, which carries the highest risk of developing HHcy, but with mild or modest effects in CC and CT genotype. Our previous studies have found that the correlation between Hcy and folate was stronger in male (CC, CT and TT) and female TT group, while the correlation of Hcy and Vit B12 was stronger in female-CC and female-CT groups (13). This give us a hint that different group might have specific correctable factors that may improve the Hcy level (9).

In this study, we found that $\mathrm{Cr}$ was another common risk factor, which was positively correlated with HHcy in all the subjects. Our findings are in accordance with previous report by Han, et al (25), which found that $\mathrm{Cr}$ was a common factor for HHcy in both healthy and hypertensive subjects. Another support for this relationship was that Hcy level could be increased in a dose-response effect by Guanidinoacetic acid (GAA), which is an intermediate in the biosynthesis of $\mathrm{Cr}(26)$.

Furthermore, we identified some specific risk factors for each gender and genotype (see Table 4). This is quite unique from the other previous studies, which targeting the whole genotype population $(12,25)$. First, we found that aging was just a risk factor for CC and CT genotypes, but not for TT genotype. Though former research has identified age as a positive risk factor for HHcy, yet it was not for all the genotypes. In fact, we observed that participants with TT genotype already had a high level of Hcy at the early age of 20 $\sim 40 \mathrm{y}$. Therefore, the intervention of decreasing Hcy should be performed at an early age for TT genotype, and it can be intervened later for CC and CT genotypes.

Table 4

Risk factors for HHcy for each gender and MTHFR C677T genotype*.

\begin{tabular}{|lllllll|}
\hline & F-CC & F-CT & F-TT & M-CC & M-CT & M-TT \\
\hline Folate & -0.119 & -0.172 & -0.453 & -0.265 & -0.412 & -0.991 \\
\hline VitB12 & -0.004 & -0.004 & -0.005 & -0.003 & -0.005 & -0.011 \\
\hline Cr & 0.083 & 0.075 & 0.124 & 0.047 & 0.08 & 0.106 \\
\hline Age & - & 0.062 & - & 0.065 & 0.038 & - \\
\hline SBP & - & 0.015 & 0.046 & - & - & 0.038 \\
\hline AST & - & - & 0.108 & 0.054 & 0.011 & 0.085 \\
\hline Hb & - & 0.027 & - & 0.032 & - & 0.087 \\
\hline *All these risk factors are significantly associated with HHcy levels $(p<0.05)$. \\
\hline
\end{tabular}


SBP is also highly correlated with HHcy, and both SBP and HHcy are independent predictors for the stroke morbidity in hypertension population (27). Data analysis from the National Health and Nutrition Examination Survey (NHANES) showed that Hcy was positively associated with SBP, and this association was stronger in women than in men (28). However, it was controversial in the Hordaland study, which showed that the correlation of Hcy with SBP was really weak, even though positive (29). In this study, we discovered that correlation SBP was positively correlated with Hcy in TT and CT genotypes, but not in CC groups for both genders (see Table 4). This suggested that gene-related HHcy may play an important role for H-type hypertension. However, it does not mean that HHcy would not influence SBP for CC group, only indicated that some other risk factors might outweigh this relationship.

AST was another positive risk factor for HHcy for male (CC, CT and TT) and female (TT). At present, the association between Hcy and AST were still controversial. Li et al. (30) have investigate the effect of MTHFR gene polymorphisms and serum Hcy and folate level on the hepatic functions in a Chinese hypertensive population, but they did not find a significant correlation between Hcy and AST. However, an inverse correlation was found between Hcy and AST in hemodialysis patients, whose remethylation of Hcy was impaired (31). The inconformity of these studies might come from the selection criteria of the subjects. A strict cohort study may clarify their associations by a much longer observation.

We also found a positive correlation between $\mathrm{Hcy}$ and $\mathrm{Hb}$ in male (CC and TT) and female (CT). At present, few studies have focus on the association of Hcy and $\mathrm{Hb}$. Schaffer A and his colleague (32) had reported that $\mathrm{Hb}$ was one of the positive risk factors for $\mathrm{HHcy}$, but further studies are still needed to confirm this relationship and explore its mechanism.

The strength of this study is that we classified people into different gender and genotype groups, both of which are strong independent variables for HHcy. There are several beneficial from this classification. First, we exclude some confound bias among groups, such as age, which showed different influential strength among groups. Second, we discovered some new variables that may influence the Hcy level, even though they still need further prospective studies to confirm.

However, there are still some limitations for this study. First, there might be more risk factors to be discovered in addition to our findings. Variables selected for this study was only the physical and laboratory examination. Other factors should be taken into consideration as they were proved to be important for Hcy metabolism, such as smoking, drinking, nutrition, physical exercise, et al. Second, the correlation between HHcy and the risk factors in this cross-section study cannot reflect the causal relationship. A well-designed cohort study or randomized clinical trial is needed to confirm the causality. Third, the result of this single-center study would be corroborated by multi-center collaborations, and it would be more authentic if the result can be verified in different centers.

In summary, we have found that except the common protective factors (folate and Vit B12) and risk factor $(\mathrm{Cr})$, each gender and genotype group has it specific risk factors for HHcy: female-CT (age, SBP and $\mathrm{Hb}$ ), female-TT (SBP and AST) male-CC (age, AST and Hb), male-CT (age and AST) and male-TT (SBP, AST and $\mathrm{Hb}$ ). These results might be useful for precise predicting or prevention of HHcy in the future. 


\section{Declarations}

\section{Acknowledgements}

YL ang QZ had full access to all of data in the study and takes responsibility for the integrity of the data and the accuracy of the data analysis. Conceptualization, Yang Li; Data curation, Tianyuan Xiang; Formal analysis, Muyang Yan; Funding acquisition, Qiang Zeng and Yang Li; Investigation, Hang Xiang; Methodology, Qilong Ying; Project administration, Hang Xiang, Qiang Zeng and Yang Li; Resources, Hang Xiang and Tianyuan Xiang; Software, Sheng Yu; Supervision, Muyang Yan and Qiang Zeng; Validation, Sheng Yu; Writing - original draft, Hang Xiang; Writing - review \& editing, Qilong Ying.

\section{Sources of Funding}

The present study was supported by the National Natural Science Foundation of China (No.81870249) and the National Natural Science Foundation of China International cooperation program 『No.81561128020区.

\section{Conflicts of interest}

All authors read and approved the final manuscript. None of the authors reported a conflict of interest related to the study.

\section{References}

1. Ravaglia G, Forti P, Maioli F, Muscari A, Sacchetti L, Arnone G, Nativio V, Talerico T, Mariani E. Homocysteine and cognitive function in healthy elderly community dwellers in Italy. The American journal of clinical nutrition. 2003;77:668-73.

2. Ueland PM, Refsum $H$. Plasma homocysteine, a risk factor for vascular disease: plasma levels in health, disease, and drug therapy. J Lab Clin Med. 1989;114:473-501.

3. Homocysteine Studies Collaboration. Homocysteine and risk of ischemic heart disease and stroke: a meta-analysis. JAMA. 2002;288:2015-22.

4. Jiang S, Li J, Zhang Y, Venners SA, Tang G, Wang Y, Li Z, Xu X, Wang B, Huo Y. Methylenetetrahydrofolate reductase C677T polymorphism, hypertension and risk of stroke: a prospective, nested case-control study. The International journal of neuroscience. 2017;127:253-60.

5. Petras M, Tatarkova Z, Kovalska M, Mokra D, Dobrota D, Lehotsky J, Drgova A. Hyperhomocysteinemia as a risk factor for the neuronal system disorders. Journal of physiology pharmacology. 2014;65:15-23.

6. Wang BR, Ou Z, Jiang T, Zhang YD, Zhao HD, Tian YY, Shi JQ, Zhou JS. Independent Correlation of Serum Homocysteine with Cerebral Microbleeds in Patients with Acute Ischemic Stroke due to LargeArtery Atherosclerosis. Journal of stroke cerebrovascular diseases. 2016;25:2746-51.

7. Rozen R. Genetic modulation of homocysteinemia. Seminars in thrombosis hemostasis. 2000;26:255-61. 
8. Finkelstein JD. Methionine metabolism in mammals. The Journal of nutritional biochemistry. 1990;1:228-37.

9. Taguchi T, Mori H, Hamada A, Yamori Y, Mori M. Serum folate, total homocysteine levels and methylenetetrahydrofolate reductase $677 \mathrm{C}>\mathrm{T}$ polymorphism in young healthy female Japanese. Asia Pacific journal of clinical nutrition. 2012;21:291-5.

10. Zappacosta B, Graziano M, Persichilli S, Di Castelnuovo A, Mastroiacovo P, lacoviello L. 5,10Methylenetetrahydrofolate reductase (MTHFR) C677T and A1298C polymorphisms: genotype frequency and association with homocysteine and folate levels in middle-southern Italian adults. Cell biochemistry function. 2014;32:1-4.

11. Rutten-Jacobs LC, Traylor M, Adib-Samii P, Thijs V, Sudlow C, Rothwell PM, Boncoraglio G, Dichgans M, Meschia J, Maguire J, Levi C, Rost NS, Rosand J, Hassan A, Bevan S, Markus HS. Association of MTHFR C677T Genotype With Ischemic Stroke Is Confined to Cerebral Small Vessel Disease Subtype. Stroke. 2016;47:646-51.

12. Wang Y, Li X, Qin X, Cai Y, He M, Sun L, Li J, Zhang Y, Tang G, Wang B, Sun N, Xu X, Liu L, Xu X, Huo Y. Prevalence of hyperhomocysteinaemia and its major determinants in rural Chinese hypertensive patients aged 45-75 years. The British journal of nutrition. 2013;109:1284-93.

13. Zeng Q, Li F, Xiang T, Wang W, Ma C, Yang C, Chen H, Xiang H. Influence of food groups on plasma total homocysteine for specific MTHFR C677T genotypes in Chinese population. Molecular nutrition food research. 2017; 61. Epub 2016 Oct 4. doi:10.1002/mnfr.201600351.

14. Ventura P, Panini R, Verlato C, Scarpetta G, Salvioli G. Hyperhomocysteinemia and related factors in 600 hospitalized elderly subjects. Metabolism. 2001;50:1466-71.

15. Liu XD, Gao B, Sun D, Shi M, Ma YY, Liu ZR, Wang B, Xu X, Xu X, Ji QH, Zhao G. Prevalence of hyperhomocysteinaemia and some of its major determinants in Shaanxi Province, China: a crosssectional study. The British journal of nutrition. 2015;113:691-8.

16. Refsum H, Ueland PM, Svardal AM. Fully automated fluorescence assay for determining total homocysteine in plasma. Clinical chemistry. 1989;35:1921-7.

17. Fiskerstrand T, Refsum H, Kvalheim G, Ueland PM. Homocysteine and other thiols in plasma and urine: automated determination and sample stability. Clinical chemistry. 1993;39:263-71.

18. Zironi E, Gazzotti T, Barbarossa A, Devicienti C, Scardilli M, Pagliuca G. Technical note: development and validation of a method using ultra performance liquid chromatography coupled with tandem mass spectrometry for determination of vitamin B12 concentrations in milk and dairy products. Journal of dairy science. 2013;96:2832-6.

19. Fekih-Mrissa N, Mrad M, Klai S, Mansour M, Nsiri B, Gritli N, Mrissa R. Methylenetetrahydrofolate reductase (C677T and $\mathrm{A} 1298 \mathrm{C}$ ) polymorphisms, hyperhomocysteinemia, and ischemic stroke in Tunisian patients. Journal of stroke cerebrovascular diseases. 2013;22:465-9.

20. Hustad S, Midttun O, Schneede J, Vollset SE, Grotmol T, Ueland PM. The methylenetetrahydrofolate reductase $677 \mathrm{C}->T$ polymorphism as a modulator of a $B$ vitamin network with major effects on homocysteine metabolism. American journal of human genetics. 2007;80:846-55. 
21. He JA, Hu XH, Fan YY, Yang J, Zhang ZS, Liu CW, Yang DH, Zhang J, Xin SJ, Zhang Q, Duan ZQ. Hyperhomocysteinaemia, low folate concentrations and methylene tetrahydrofolate reductase C677T mutation in acute mesenteric venous thrombosis. European journal of vascular endovascular surgery. 2010;39:508-13.

22. Huh HJ, Chi HS, Shim EH, Jang S, Park CJ. Gene-nutrition interactions in coronary artery disease: correlation between the MTHFR C677T polymorphism and folate and homocysteine status in a Korean population. Thrombosis research. 2006;117:501-6.

23. Ganji V, Kafai MR. Frequent consumption of milk, yogurt, cold breakfast cereals, peppers, and cruciferous vegetables and intakes of dietary folate and riboflavin but not vitamins B-12 and B-6 are inversely associated with serum total homocysteine concentrations in the US population. The American journal of clinical nutrition. 2004;80:1500-7.

24. Kelly P, McPartlin J, Goggins M, Weir DG, Scott JM. Unmetabolized folic acid in serum: acute studies in subjects consuming fortified food and supplements. The American journal of clinical nutrition. 1997;65:1790-5.

25. 10.1016/j.clnu.2016.11.011

Han L, Liu Y, Wang C, Tang L, Feng X, Astell-Burt T, Wen Q, Duan D, Lu N, Xu G, Wang K, Zhang L, Gu K, Chen S, Ma J, Zhang T, You D, Duan S. Determinants of hyperhomocysteinemia in healthy and hypertensive subjects: A population-based study and systematic review. Clinical nutrition (Edinburgh, Scotland). 2016. [Epub ahead of print]. doi: 10.1016/j.clnu.2016.11.011.

26. Ostojic SM, Stojanovic M, Drid P, Hoffman JR. Dose-response effects of oral guanidinoacetic acid on serum creatine, homocysteine and B vitamins levels. European journal of nutrition. 2014;53:1637-43.

27. Pang H, Han B, Fu Q, Zong Z. Association of High Homocysteine Levels With the Risk Stratification in Hypertensive Patients at Risk of Stroke. Clinical therapeutics. 2016;38:1184-92.

28. Lim U, Cassano PA. Homocysteine and blood pressure in the Third National Health and Nutrition Examination Survey, 1988-1994. American journal of epidemiology. 2002;156:1105-13.

29. Nygård O, Vollset SE, Refsum H, Stensvold I, Tverdal A, Nordrehaug JE, Ueland M, Kvåle G. Total plasma homocysteine and cardiovascular risk profile. The Hordaland Homocysteine Study JAMA. 1995;274:1526-33.

30. Li WX, Li W, Cao JQ, Yan H, Sun Y, Zhang H, Zhang Q, Tang L, Wang M, Huang JF, Liu D. Folate Deficiency Was Associated with Increased Alanine Aminotransferase and Glutamyl Transpeptidase Concentrations in a Chinese Hypertensive Population: A Cross-Sectional Study. Journal of nutritional science vitaminology. 2016;62:265-71.

31. Huang JW, Yen CJ, Pai MF, Wu KD, Tsai TJ, Hsieh BS. Association between serum aspartate transaminase and homocysteine levels in hemodialysis patients. American journal of kidney diseases. 2002;40:1195-201.

32. Schaffer A, Verdoia M, Cassetti E, Marino P, Suryapranata H, De Luca G. Novara Atherosclerosis Study Group (NAS). Relationship between homocysteine and coronary artery disease. Results from a large prospective cohort study. Thrombosis research. 2014;134:288-93. 
Figures
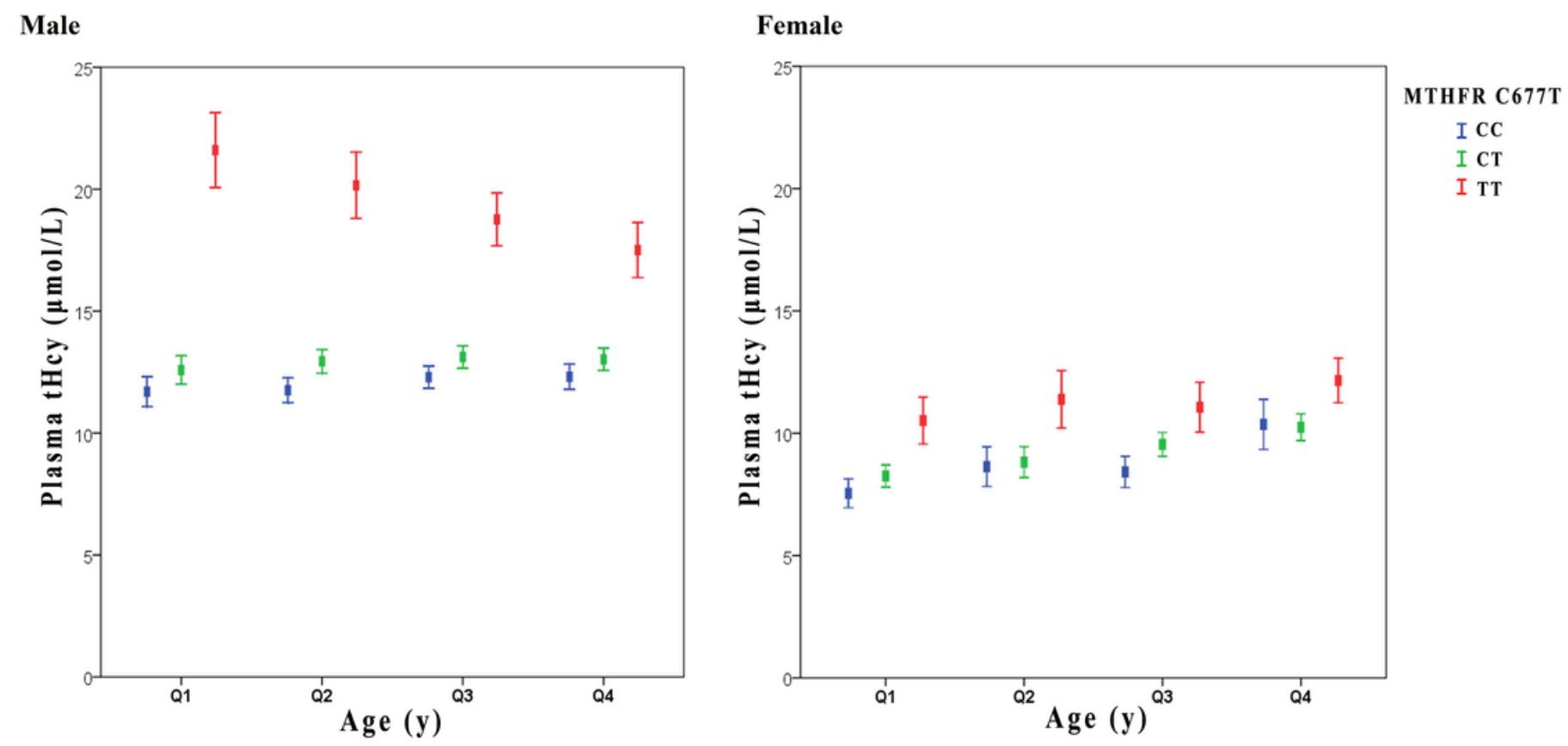

Figure 1

Figure 1

\section{Supplementary Files}

This is a list of supplementary files associated with this preprint. Click to download.

- supplementarytalbelll.docx 